University of Louisville

ThinkIR: The University of Louisville's Institutional Repository

Electronic Theses and Dissertations

$8-2020$

\title{
Linear methods for regression with small sample sizes relative to the number of variables.
}

Rajesh Sikder

University of Louisville

Follow this and additional works at: https://ir.library.louisville.edu/etd

Part of the Applied Statistics Commons, Biostatistics Commons, and the Other Applied Mathematics Commons

\section{Recommended Citation}

Sikder, Rajesh, "Linear methods for regression with small sample sizes relative to the number of variables." (2020). Electronic Theses and Dissertations. Paper 3520.

https://doi.org/10.18297/etd/3520

This Master's Thesis is brought to you for free and open access by ThinkIR: The University of Louisville's Institutional Repository. It has been accepted for inclusion in Electronic Theses and Dissertations by an authorized administrator of ThinkIR: The University of Louisville's Institutional Repository. This title appears here courtesy of the author, who has retained all other copyrights. For more information, please contact thinkir@louisville.edu. 
LINEAR METHODS FOR REGRESSION WITH SMALL SAMPLE SIZES RELATIVE TO THE NUMBER OF VARIABLES

\author{
By \\ Rajesh Sikder \\ B.Sc., Khulna University, Bangladesh

\begin{abstract}
A Thesis
Submitted to the Faculty of the

College of Arts and Sciences of the University of Louisville

in Partial Fulfillment of the Requirements

for the Degree of
\end{abstract} \\ Master of Arts in Mathematics \\ Department of Mathematics \\ University of Louisville \\ Louisville, KY
}

August 2020 



\title{
LINEAR METHODS FOR REGRESSION WITH SMALL SAMPLE SIZES RELATIVE TO THE NUMBER OF VARIABLES
}

\author{
Submitted by \\ Rajesh Sikder
}

A Thesis Approved on

August 11, 2020

by the Following Thesis Committee:

Professor Ryan Gill, Thesis Director

Department of Mathematics

Professor Dan Han

Department of Mathematics

Professor Jeremy Gaskins

Department of Bioinformatics and

Biostatistics 


\section{DEDICATION}

TO MY PARENTS 


\section{ACKNOWLEDGEMENTS}

First and foremost, I would like to thank my advisor, Professor Ryan Gill, the nicest professor I have ever seen. His office door was always open to me whenever I had any doubt about my research or writing, even in the weekend. Without him I could not make this far.

I would also like to acknowledge Professor Dan Han as one of the committee members, and I am gratefully indebted not only for her advice on this thesis but also for her valuable suggestion about my future career.

I would also like to thank Professor Jeremy Gaskins for being my thesis committee member.

Finally, I would like to thank my parents for their unconditional love, continuous support, and belief in me. 


\title{
ABSTRACT \\ LINEAR METHODS FOR REGRESSION WITH SMALL SAMPLE SIZES RELATIVE TO THE NUMBER OF VARIABLES
}

\author{
Rajesh Sikder
}

August 11, 2020

In data sets where there are a small number of observations but a large number of variables observed for each observation, ordinary least squares estimation cannot be used for regression models. There are many alternative including stepwise regression, penalized methods such as ridge regression and the LASSO, and methods based on derived inputs such as principal components regression and partial least squares regression. In this thesis, these five methods are described. $K$-fold cross validation is also discussed as a way for determining regularization parameters for each method. The performance of these methods in estimation and prediction is also examined through simulation studies under various interesting scenarios. Finally, the methods will be applied to a real data set in which each method is applied to build a model for the weights of mice based on microarray expression data for a large number of genes. 


\section{TABLE OF CONTENTS}

\section{CHAPTER}

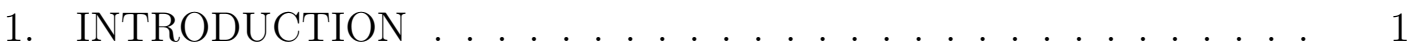

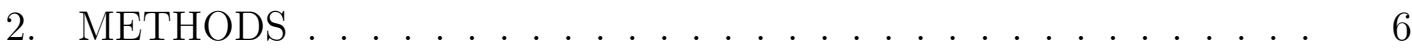

2.1 FORWARD SELECTION $\ldots \ldots \ldots \ldots \ldots$

2.2 RIDGE REGRESSION $\ldots \ldots \ldots \ldots \ldots$

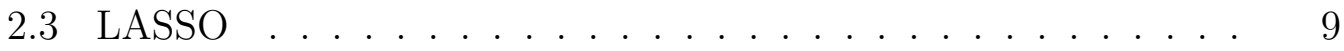

2.4 PRINCIPAL COMPONENTS REGRESSION . . . . . . . . . 10

2.5 PARTIAL LEAST SQUARES REGRESSION . . . . . . . . . 12

2.6 LONGLEY EXAMPLE . . . . . . . . . . . . . . . . . . . . 13

3. SIMULATION STUDIES . . . . . . . . . . . . . . . . 23

3.1 SCENARIO 1 WITH $p=10, G=4, \rho=.1, \beta_{1}=.1 \ldots \ldots 24$

3.2 SCENARIO 1 WITH $G=4, \rho=.1, \beta_{1}=.5, \sigma=.1 \ldots 30$

3.3 SCENARIO 2 WITH $p=10, G=4, \rho=.1 \ldots \ldots$

3.4 SCENARIO 2 WITH $G=4, \rho=.1, \sigma=.01 \ldots \ldots \ldots$

4. REAL DATA ......................... 37

4.1 DATASET BACKGROUND AND PREPROCESSING . . . . 37

4.2 MODEL BUILDING AND RESULTS $\ldots \ldots \ldots \ldots$

4.3 COMPARISON OF RESULTS . . . . . . . . . . . . 40

REFERENCES . . . . . . . . . . . . . . . . . . . . 42

CURRICULUM VITAE . . . . . . . . . . . . . . . . . . . 44 


\section{LIST OF TABLES}

Table 3.1. Estimation and predictive errors under scenario 1 when $p=10$, $G=4, \rho=.10, \beta_{1}=.1, \sigma=.01 \ldots \ldots \ldots \ldots \ldots 28$

Table 3.2. Estimation and predictive errors under scenario 1 when $p=10$, $G=4, \rho=.10, \beta_{1}=.1, \sigma=.1 \ldots \ldots \ldots \ldots$ 


\section{LIST OF FIGURES}

Figure 2.1. Computing the LASSO estimates for the Longley data example. 17

Figure 3.1. Estimation and predictive errors under scenario 1 when $p=10$, $G=4, \rho=.10, \beta_{1}=.1 \ldots \ldots \ldots \ldots$

Figure 3.2. Estimation and predictive errors under scenario 1 when $G=4$, $\rho=.1, \beta_{1}=.5, \sigma=.1 \ldots \ldots \ldots \ldots$

Figure 3.3. Estimation and predictive errors under scenario 2 when $p=10$, $G=4, \rho=.10 \ldots \ldots \ldots \ldots$

Figure 3.4. Estimation and predictive errors under scenario 2 when $G=4$, $\rho=.10, \sigma=.01 \ldots \ldots \ldots \ldots \ldots \ldots$ 


\section{CHAPTER 1 INTRODUCTION}

Regression analysis is a statistical method used to relate a variable of interest, typically denoted by $y$, the dependent variable, to a set of independent variables, denoted by say $x_{1}, x_{2}, \ldots, x_{p}$. The goal is to build a model that assists statisticians in describing, controlling, and predicting the dependent variable based on the independent variable(s). There are many types of regression analysis: Simple and Multiple Linear Regression and Nonlinear Regression to name a few.

In this thesis, we consider linear methods for regression. Given a data set $\left(x_{1,1}, \ldots, x_{p, 1}, y_{1}\right), \ldots,\left(x_{1, n}, \ldots, x_{p, n}, y_{n}\right)$ to train the model, the multiple linear regression model assumes that

$$
y_{i}=\beta_{0}+\sum_{j=1}^{p} \beta_{j} x_{j, i}+\epsilon_{i}
$$

for $i=1, \ldots, n$ and $j=1 \ldots, p$ where the errors $\epsilon_{1}, \ldots, \epsilon_{n}$ are independent random variables with mean 0 and variance $\sigma^{2}$. The simplest method for fitting this model is least squares estimation in which estimates $\hat{\beta}_{0}, \hat{\beta}_{1}, \ldots, \hat{\beta}_{p}$ are obtained by finding the values which minimize the residual sum of squares function

$$
R S S\left(b_{0}, b_{1}, \ldots, b_{p}\right)=\sum_{i=1}^{n}\left(y_{i}-b_{0}+\sum_{j=1}^{p} b_{j} x_{j, i}\right)^{2} .
$$

Here the minimum value of this function is $\sum_{i=1}^{n} e_{i}^{2}$ where $e_{i}=y_{i}-\hat{y}_{i}$ is the $i$ th residual based on the $i$ th fitted value $\hat{y}_{i}=\hat{\beta}_{0}+\sum_{j=1}^{p} \hat{\beta}_{j} x_{j, i}$. That is, the sum of squared distances between the regression surface and the data points is minimal. Note that the residuals are not independent since they are constrained so that $\sum_{i=1}^{n} e_{i}=0[14]$. 
The slopes of their individual explanatory variables are the constants $\beta_{1}, \ldots, \beta_{p}$, and are referred to as coefficients of the variables. For instance, $\beta_{j}$ is the change in the predicted value of $y$ per unit of change in $x_{j}$, while holding the other variables constant. The additional unknown coefficient $\beta_{0}$, known as intercept, is the mean for the response if all the $x$ 's are zero (in some models this input point might not be meaningful). The coefficients and intercept are estimated by least squares, i.e., setting them equal to the unique values that minimize the sum of squared errors within the sample of data to which the model is fitted. And the model's prediction errors are often assumed to be independently and identically normally distributed.

Now, let's say $n$ is the number of observations (data point) and $p$ is the number of variables (feature) as discussed above. There can be the following three cases:

- when $n$ is much larger than $p$, i.e., number of observation $>$ number of variables. In this case, the least squares estimates tend to also have low variance, and hence will perform well on test observations.

- when $n$ is not much larger than $p$, i.e., number of observation $\sim=$ number of variables, there can be a lot of variability in the least squares fit, resulting in overfitting and consequently poor predictions on future observations not used in model training.

- when $p$ is larger than $n$ i.e., number of observation $<$ number of variables, there is no longer a unique least squares coefficient estimate: the variance is infinite so the method cannot be used at all [1].

The formula for computing the least squares estimator of the vector of regression coefficients $\beta=\left[\beta_{0}, \beta_{1}, \ldots, \beta_{p}\right]^{t}$ can be expressed in matrix notation as

$$
\hat{\beta}_{l s}=\left(X^{t} X\right)^{-1} X^{t} y
$$


where

$$
X=\left[\begin{array}{cccc}
1 & x_{1,1} & \cdots & x_{p, 1} \\
1 & x_{1,2} & \cdots & x_{p, 2} \\
\vdots & \vdots & \ddots & \vdots \\
1 & x_{1, n} & \cdots & x_{p, n}
\end{array}\right]
$$

and

$$
y=\left[\begin{array}{c}
y_{1} \\
y_{2} \\
\vdots \\
y_{n}
\end{array}\right]
$$

However, this formula can only be computed when the inverse of $X^{t} X$ exists. As shown in [14], the inverse of $X^{t} X$ is less than or equal to $\min \{n, p+1\}$. But if $n<p+1$, this rank is less than $p+1$ but $X^{t} X$ is a $(p+1) \times(p+1)$ square matrix. If a square matrix is not full rank, then its determinant is 0 which allows implies that it is not invertible [11]. So, in this case, we must consider alternatives to Least Squares Estimation to try to fit the model.

As an example, we look the Longley data set [12] using the response Employed and two explanatory variables Population and GNP. 
The design matrix and response vector for this analysis are

$$
X=\left[\begin{array}{ccc}
1 & 107.608 & 234.289 \\
1 & 108.632 & 259.426 \\
1 & 109.773 & 258.054 \\
1 & 110.929 & 284.599 \\
1 & 112.075 & 328.975 \\
1 & 113.270 & 346.999 \\
1 & 115.094 & 365.385 \\
1 & 116.219 & 363.112 \\
1 & 117.388 & 397.469 \\
1 & 118.734 & 419.180 \\
1 & 120.445 & 442.769 \\
1 & 121.950 & 444.546 \\
1 & 123.366 & 482.704 \\
1 & 125.368 & 502.601 \\
1 & 127.852 & 518.173 \\
1 & 130.081 & 554.894
\end{array}\right] \text { and } y=\left[\begin{array}{l}
60.323 \\
61.122 \\
60.171 \\
61.187 \\
63.221 \\
63.639 \\
64.989 \\
63.761 \\
66.019 \\
67.857 \\
68.169 \\
66.513 \\
68.655 \\
69.564 \\
69.331 \\
70.551
\end{array}\right]
$$

respectively.

The least squares estimate of $\beta$ based on these variables is

$$
\hat{\beta}_{l s}=\left(X^{t} X\right)^{-1} X^{t} y=\left[\begin{array}{c}
88.9388 \\
-0.4097 \\
0.0632
\end{array}\right] \text {. }
$$

In this thesis, we will study when the number of variables is much greater than observations, i.e., small n-large-p, so as we have seen, Least Squares Estimation for linear regression would not work. That's why we introduce in chapter 2, Stepwise regression (Forward Selection), Ridge, LASSO, Principal Components Regression 
and Partial Least Squares Regression, which at least have the potential to solve this kind of problem. In chapter 3, we will study the performance of these methods in estimation and prediction through simulation studies under different scenarios. In chapter 4, we will study the methods applied to a real data set to build a model for the weights of mice based on microarray expression data for a large number of genes. 


\section{CHAPTER 2}

\section{METHODS}

In this section, we will discuss the five methods, Forward Selection, Ridge Regression, LASSO, Principal Components Regression and Partial Least Squares Regression. In later chapters, the performance of these methods will be examined in simulation studies and also in real data where we predict the weight of mice based on gene expression intensities.

\subsection{FORWARD SELECTION}

A natural modification to least squares estimation is to try to select a subset of variables and then use the least squares criteria to estimate the parameters for the variables in that subset, setting the coefficient estimates for all other variables equal to 0 . Even when $n$ is large relative to $p$, this can help increase prediction accuracy by trading an increase in bias for a decrease in variance and help with interpretation by producing a more parsimonious model even if it sacrifices some details [7]. In that setting, variable selection by choosing the best subset which minimizes some pre-determined criteria such as the Akaike information criterion (AIC) or the Bayesian information criterion (BIC) seems like a natural approach

for variable selection. A description of best subset regression and some stepwise procedures are described in [16]. Other criteria such as cross validation, p-values, the $C_{p}$ statistic [2], etc.

However, when $p$ is large, then the $2^{p}$ possible models that need to be consid- 
ered is not computationally tractable. In these situations, greedy algorithms such as forward selection and backward elimination are commonly used. Forward selection begins with only an intercept and then adds variables one by one. At each step, the variable that is added to the model which corresponds to the largest decrease in the sum of the squared residuals. Then, a pre-determined criteria is used to choose among the path of models encountered by the forward selection procedure. Forward selection can be stopped the first time the added variable does not lead to an improvement in the pre-determined criteria, it can examine an entire path of models encountered, or it can do something in between these options.

In typical situations where the sample size $n$ is larger than $p$, backward elimination is an option. In backward elimination, we instead start with all variables included in the model, and then eliminate variables one by one by removing the variable which corresponds to a model with the smallest increase in the sum of the squared residuals. Then a similar process could be used to determine the optimal number of variables based on some pre-determined criteria.

However, when $n<p+1$, any linear combination of $n$ independent explanatory variables will lead to a perfect fit where the sum of squared residuals equals 0 . So, backward elimination cannot be used in the setting where $p$ is much larger than $n$ since dropping most variables will still lead to a model with a perfect fit. So, in this setting we are interested in, we will only study forward selection where the number of variables included in the model is the regularization parameter.

\subsection{RIDGE REGRESSION}

Ridge regression is commonly used when multicollinearity is present in linear regression, which is common in models with large numbers of parameters. In general, the method tries to provide improved efficiency in parameter estimation 
problems in exchange for biasing the parameter estimates towards 0.

In ridge regression, the parameter estimates depend on the way the inputs are scaled, so typically the input variables are standardized by subtracting their means and dividing by their standard deviations. To avoid notational issues, we assume in this section that the design matrix $X$ does not include an intercept and its columns have been standardized and the response vector $y$ has been centered [7]. In the end, the estimates can be converted back to the original scale.

The modification that leads to the ridge regression estimates is to add a penalty term to the residual sum of squares to obtain a penalized residual sum of squares function

$$
\operatorname{PRSS}\left(b_{1}, \ldots, b_{p}, \lambda\right)=\sum_{i=1}^{n}\left(y_{i}-\sum_{j=1}^{p} b_{j} x_{j, i}\right)^{2}+\lambda \sum_{j=1}^{p} b_{j}^{2} .
$$

Note that the intercept has been excluded since the explanatory variables are assumed to be standardized and the responses are assumed to be centered. This is equivalent to the dual problem of minimizing $R S S\left(b_{1}, \ldots, b_{p}\right)$ subject to the constraint

$$
\sum_{j=1}^{p} b_{j}^{2} \leq T
$$

for some $T$. When the constraint is not satisfied, there is a one-to-one correspondence between $\lambda$ and $T$ as shown in [10].

Considering $\lambda$ to be fixed, $P R S S$ can be minimized as a function of $b_{1}, \ldots, b_{p}$ and the vector of ridge regression estimates can be obtained by the formula

$$
\hat{\beta}_{r r}=\left(X^{t} X+\lambda I_{p}\right)^{-1} X^{t} y
$$

which as seen in the formula adds a small value $\lambda$ to the diagonal elements of $X^{t} X$ so that the resulting matrix is invertible. More general expressions where some of the coefficients (such as the intercept) are not penalized can be formulated as shown in $[10]$. 
For any value of $\lambda$, the inverse will exist so these estimator is well-defined even when $p$ is much larger than $n$. The estimator $\hat{\beta}_{r r}$ is biased towards 0 as shown by the formula

$$
E\left(\hat{\beta}_{r r}-\beta\right)=\left[\left(X^{t} X+\lambda I_{p}\right)^{-1} X^{t} X-I_{p}\right] \beta
$$

and its covariance matrix is given by

$$
\operatorname{Var}\left(\hat{\beta}_{r r}\right)=\left(X^{t} X+\lambda I_{p}\right)^{-1} X^{t} X\left(X^{t} X+\lambda I_{p}\right)^{-1}
$$

It can be shown that there does exist a value of $\lambda$ for which the mean squared error of the ridge estimator is less than that of the least squares estimator [15], but unfortunately, the appropriate value of $\lambda$ depends on the true underlying regression coefficients and so there is not an analytic solution for the optimality of the ridge solution [1]. So, $\lambda$ is considered a regularization parameter that is selected based on a pre-determined criteria such as cross-validation.

\subsection{LASSO}

The LASSO is another penalized regression method. The name is an acronym which stands for Least Absolute Shrinkage and Selection Operator. Instead of imposing the penalty term based on the squared coefficients, the LASSO instead penalizes models where the absolute value of the magnitude of the coefficients is large. In other words, the LASSO performs L1 regularization.

As in ridge regression, the parameter estimates depend on the way the inputs are scaled, so typically the input variables are standardized by subtracting their means and dividing by their standard deviations. To avoid notational issues, we assume in this section that the design matrix $X$ does not include an intercept and its columns have been standardized and the response vector $y$ has been centered [7]. In the end, the estimates can be converted back to the original scale. This conversion is shown for the LASSO in [9]. 
So the LASSO minimizes a different penalized residual sum of squares function

$$
\operatorname{PRSS}_{2}\left(b_{1}, \ldots, b_{p}, \lambda\right)=\sum_{i=1}^{n}\left(y_{i}-\sum_{j=1}^{p} b_{j} x_{j, i}\right)^{2}+\lambda \sum_{j=1}^{p}\left|b_{j}\right| .
$$

Minimizing $P R S S_{2}$ as a function of $b_{1}, \ldots, b_{p}$ is equivalent to the dual problem of minimizing $R S S$ subject to the constraint

$$
\sum_{j=1}^{p}\left|b_{j}\right| \leq T
$$

for some $T$. For small $p$ this can be easily computed since it is a quadratic programming problem. But, for larger $p$, there is a more complicated algorithm known as Least Angle Regression [4] which helps to efficiently compute the estimates which minimize $P R S S_{2}$ as a function of $b_{1}, \ldots, b_{p}$.

An important distinction between the LASSO and ridge regression is that the LASSO naturally produces parameter estimates which include some coefficients which are 0 and thus the corresponding variables are not included in the model. On the other hand, ridge regression does not eliminate variables in this manner because of the shape of the boundary of its constraint (for the LASSO the boundary is diamond shaped with vertices on the coefficient axes whereas for ridge regression the boundary is spherical). If $n<p+1$, the LAR algorithm used in the LASSO has a solution where $R S S=0$ so it will include at most $n-1$ variables.

The regularization or tuning parameter $\lambda$ controls the sparcity of the model. As $\lambda$ increases from 0 to $\infty$, the bias increases and the variance decreases as we also saw with ridge regression, but more variables are eliminated from the model, approaching a model for the response only based on the intercept. The parameter of $\lambda$ is selected based on a pre-determined criteria such as cross-validation.

\subsection{PRINCIPAL COMPONENTS REGRESSION}

Principal Components Regression is a method which applies the least squares 
estimate to a smaller set of $k$ derived inputs. So, when $p>n$, it simply creates new inputs which are linear combinations of the old inputs and uses them in a linear regression model.

As in ridge regression and the LASSO, the parameter estimates depend on the way the inputs are scaled, so typically the input variables are standardized by subtracting their means and dividing by their standard deviations. To avoid notational issues, we assume in this section that the design matrix $X$ does not include an intercept and its columns have been standardized and the response vector $y$ has been centered. In the end, the estimates can be converted back to the original scale.

Now, the $j$ th principal components direction $v_{j}$ is the eigenvector corresponding to the $j$ th largest eigenvalue of $X^{t} X$. Then the $j$ th principal component is defined to be the vector $z_{j}=X v_{j}$. The largest principal component maximizes the variance of the projected data, and each subsequent principal component maximize the variance subject to being orthogonal to the previous principal components [7]. Even when $n>p$, this method is designed to handle multicollinearity by adding some bias by removing information to reduce the standard errors.

Then Principal Components Regression uses the design matrix $Z$ with columns $z_{1}, \ldots, z_{k}$ and uses the least squares estimate to obtain coefficients estimates

$$
\hat{\theta}_{p c r}=\left(Z^{t} Z\right)^{-1} Z^{t} y
$$

in the derived input space. Since the columns of $Z$ are linear combinations of the columns of $X$, the Then the estimate of the regression coefficients is

$$
\hat{\beta}_{p c r}=V \hat{\theta}_{p c r}
$$

where $V$ is a matrix with the eigenvectors as columns. Note that $V$ is orthogonal.

Since the variables are standardized, it can be written that $X^{t} X=R$, where $R$ is the sample correlation matrix of the explanatory variables. The formula for 
Principal Components Regression can be simplified using the fact that we can write $X^{t} X=V D V^{t}=Z^{t} Z$ where $D$ is a diagonal matrix with diagonal elements corresponding to the eigenvalues of $X^{t} X$.

So, the regularization or tuning parameter here is $k$, the number of principal components. In practice, often this is selected once a pre-specified percentage of the variation in the input space has been explained. Or, it can be selected by cross-validation as we will do in our simulations and real data analysis.

\subsection{PARTIAL LEAST SQUARES REGRESSION}

Partial Least Squares Regression is another method which applies least squares estimates to a smaller set of variables derived from the original set of explanatory variables. However, instead of only using information about the variation in directions in the input space, the idea behind Partial Least Squares Regression is to also utilize information about the response $y$ to help chose the derived input variables.

As in the other methods, the parameter estimates depend on the way the inputs are scaled, so typically the input variables are standardized by subtracting their means and dividing by their standard deviations. To avoid notational issues, we assume in this section that the design matrix $X$ does not include an intercept and its columns have been standardized and the response vector $y$ has been centered. In the end, the estimates can be converted back to the original scale.

First, the columns of $X$ are weighted by regressing each column $x_{j}$ on the response $y$ to obtain weights $\hat{\phi}_{1 j}=\left(x_{j}^{t} y\right) /\left(x_{j}^{t} x_{j}\right)$ for the first partial least squares

direction $z_{1}=\sum_{j=1}^{p} \hat{\phi}_{1 j} x_{j}$. Then each $x_{j}$ is orthogonalized with respect to $z_{1}$ using the formula

$$
x_{j}^{(1)}=x_{j}-\frac{z_{1}^{t} x_{j}}{z_{1}^{t} z_{1}} z_{1} .
$$

Subsequent partial least squares directions $z_{m}$ are obtained in a similar way using 
$x_{j}^{(m)}$ instead of $x_{j}$. Similar to principal components regression, the least squares estimate using the design matrix $\mathrm{Z}$ with partial least squares directions as its components is

$$
\hat{\theta}_{p l s r}=\left(Z^{t} Z\right)^{-1} Z^{t} y \text {. }
$$

Then since each column of $Z$ is a linear combination of the columns of $X$, the Partial Least Squares Regression coefficient estimates $\hat{\beta}_{p l s r}$ can be obtained by solving a system of linear equations [7].

Mathematically, it turns out that the $j$ th partial least squares direction is the

unit vector $\hat{\delta}_{j}$ such that $\operatorname{Cor}^{2}(y, X \delta) \operatorname{Var}(X \delta)$ is maximized subject to the condition that $\delta$ is orthogonal to $S \hat{\delta}_{m}$ for $m=1, \ldots, j-1$. So, it finds directions for which the projected inputs have high variance and also are highly correlated with the response.

The regularization or tuning parameter here is $k$, the number of partial least squares directions. Again, this can be selected by cross validation, among other ways.

\subsection{LONGLEY EXAMPLE}

Now we apply these these methods on small dataset to show how these methods work.

Let's see how forward selection picks the first variable to use with the Longley data. 
Let,

$$
X_{1}=\left[1, x_{1}\right]=\left[\begin{array}{cc}
1 & 107.608 \\
1 & 108.632 \\
1 & 109.773 \\
1 & 110.929 \\
1 & 112.075 \\
1 & 113.270 \\
1 & 115.094 \\
1 & 116.219 \\
1 & 117.388 \\
1 & 118.734 \\
1 & 120.445 \\
1 & 121.950 \\
1 & 123.366 \\
1 & 125.368 \\
1 & 127.852 \\
1 & 130.081
\end{array}\right] \text { and } X_{2}=\left[1, x_{2}\right]=\left[\begin{array}{cc}
1 & 234.289 \\
1 & 259.426 \\
1 & 258.054 \\
1 & 284.599 \\
1 & 328.975 \\
1 & 346.999 \\
1 & 365.385 \\
1 & 363.112 \\
1 & 397.469 \\
1 & 419.180 \\
1 & 442.769 \\
1 & 444.546 \\
1 & 482.704 \\
1 & 502.601 \\
1 & 518.173 \\
1 & 554.894
\end{array}\right]
$$

where $x_{1}$ and $x_{2}$ are column vectors representing the vectors of values for Population and $G N P$, respectively, and $X_{1}$ and $X_{2}$ are design matrices for a regression model with an intercept and the respective variable indicated by the index.

The residual sum of squares for the model using only Population as an explanatory variable is

$$
R S S_{1}=y^{t}\left(I-X_{1}\left(X_{1}^{t} X_{1}\right)^{-1} X_{1}^{t}\right) y=14.3659
$$

and the residual sum of squares for the model using only GNP as an explanatory variable is

$$
R S S_{2}=y^{t}\left(I-X_{2}\left(X_{2}^{t} X_{2}\right)^{-1} X_{2}^{t}\right) y=6.0361 .
$$


Since $R S S_{2}<R S S_{1}$ and $\left(X_{2}^{t} X_{2}\right)^{-1} X_{2}^{t} y=\left[\begin{array}{c}51.8436 \\ 0.0348\end{array}\right]$, the estimate based on the first variable added in forward selection is $\hat{\beta}_{f s}=\left[\begin{array}{c}51.8436 \\ 0 \\ 0.0348\end{array}\right]$.

Now, the design matrix need to be standardized and the response vector should be centered. The design matrix and response vector for this analysis are

$$
X_{s}=\left[\begin{array}{rr}
-1.411135233 & -1.5434331 \\
-1.263926342 & -1.2905329 \\
-1.099897684 & -1.3043364 \\
-0.933712647 & -1.0372705 \\
-0.768965196 & -0.5908091 \\
-0.597173570 & -0.4094719 \\
-0.334957732 & -0.2244927 \\
-0.173229213 & -0.2473611 \\
-0.005175313 & 0.0983004 \\
0.188323875 & 0.3167321 \\
0.434294982 & 0.5540580 \\
0.650651800 & 0.5719362 \\
0.854214095 & 0.9558390 \\
1.142018979 & 1.1560203 \\
1.499115547 & 1.3126882 \\
1.819553652 & 1.6821336
\end{array}\right] \text { and } y_{c}=\left[\begin{array}{r}
-4.994 \\
-4.195 \\
-5.146 \\
-4.130 \\
-2.096 \\
-1.678 \\
-0.328 \\
-1.556 \\
0.702 \\
2.540 \\
2.852 \\
1.196 \\
3.338 \\
4.247 \\
4.014 \\
5.234
\end{array}\right]
$$

respectively.

Now, we consider ridge regression with the penalty fixed to be $\lambda=0.1429294$. This penalty is equivalent to the penalty selected by cv.glmnet (though the glmnet 
package uses a different scale for the penalty). The ridge regression estimate on the scaled/centered data is

$$
\hat{\theta}_{r r}=\left(X_{s}^{t} X_{s}+\lambda I_{2}\right)^{-1} X_{s}^{t} y_{c}=\left[\begin{array}{c}
-0.4994887 \\
3.9119648
\end{array}\right]
$$

Dividing each component by the standard deviation of the corresponding explanatory variable $s_{j}=\sqrt{\frac{1}{15} \sum_{i=1}^{16}\left(x_{j, i}-\bar{x}_{j}\right)^{2}}$,

$$
\begin{gathered}
\hat{\beta}_{1, r r}=\theta_{1, r r} / s_{1}=-0.07180584, \\
\hat{\beta}_{2, r r}=\hat{\theta}_{2, r r} / s_{2}=0.03935779
\end{gathered}
$$

and the estimate of the intercept is

$$
\bar{y}-\hat{\beta}_{1, r r} \bar{x}_{1}-\hat{\beta}_{2, r r} \bar{x}_{2}=58.48978
$$

so,

$$
\hat{\beta}_{r r}=\left[\begin{array}{c}
58.4898 \\
-0.0718 \\
0.0394
\end{array}\right] .
$$

The LASSO also uses standardized inputs and centered outputs. We consider the LASSO with the penalty fixed to be $\lambda=0.5069551$. This penalty is equivalent to the penalty selected by cv.glmnet (though the glmnet package uses a different scale for the penalty).

To compute the LASSO estimator directly, compute the least squares estimator

$$
\hat{\theta}_{l s}=\left(X_{s}^{t} X_{s}\right)^{-1} X_{s}^{t} y_{c}=\left[\begin{array}{c}
-2.850213 \\
6.279020
\end{array}\right]
$$

for the standardized/centered data. This point is in the upper part above the red line in the second quadrant as illustrated in Figure 2.1. So it must lie on the 
Figure 2.1-Computing the LASSO estimates for the Longley data example.

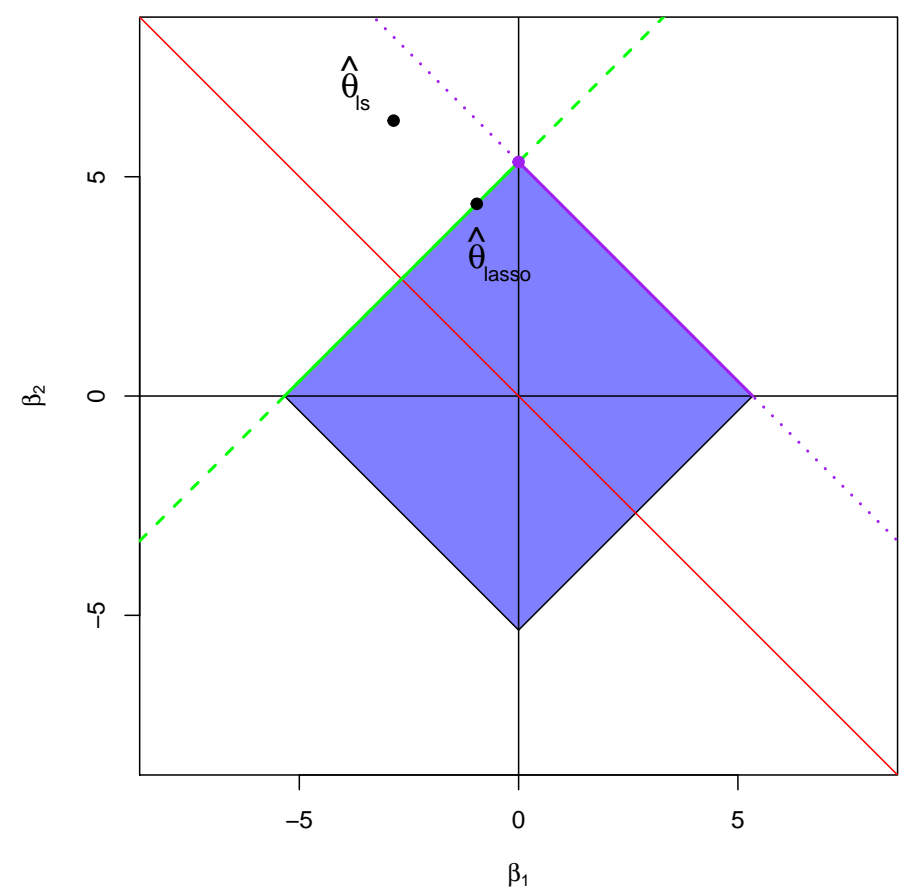

upper left boundary (green line segment) or the upper right boundary (purple line segment) on the diamond.

If it is on the green line segment, then $\beta_{1}<0$ and $\beta_{2}>0$. In this case, the form of $P R S S_{2}$ is

$$
\operatorname{PRSS}_{2}\left(b_{1}, b_{2}, \lambda\right)=\sum_{i=1}^{n}\left(y_{i}-b_{1} x_{1, i}-b_{2} x_{2, i}\right)^{2}+\lambda\left(-b_{1}+b_{2}\right) .
$$

and the minimizer of $P R S S_{2}$ is

$$
\left(X_{s}^{t} X_{s}\right)^{-1} X_{s}^{t} y_{c}-\frac{\lambda}{2}\left[\begin{array}{c}
-1 \\
1
\end{array}\right]=\left[\begin{array}{c}
-0.9536213 \\
4.3824283
\end{array}\right]
$$

which is in the second quadrant.

If the minimizer is on the purple line segment, then $\beta_{1}>0$ and $\beta_{2}>0$. In this case, the form of $P R S S_{2}$ is

$$
\operatorname{PRSS}_{2}\left(b_{1}, b_{2}, \lambda\right)=\sum_{i=1}^{n}\left(y_{i}-b_{1} x_{1, i}-b_{2} x_{2, i}\right)^{2}+\lambda\left(b_{1}+b_{2}\right) .
$$


and the minimizer of $P R S S_{2}$ is

$$
\left(X_{s}^{t} X_{s}\right)^{-1} X_{s}^{t} y_{c}-\frac{\lambda}{2}\left[\begin{array}{l}
1 \\
1
\end{array}\right]=\left[\begin{array}{c}
-2.858700 \\
6.270533
\end{array}\right]
$$

which is not in the first quadrant so the minimizer on the line segment is at the closest corner. Since that corner is also on the green line segment, the LASSO estimator is the minimizer of the green line segment

$$
\hat{\theta}_{\text {lasso }}=\left[\begin{array}{c}
-2.858700 \\
6.270533
\end{array}\right] \text {. }
$$

Dividing each component by the standard deviation of the corresponding explanatory variable $s_{j}=\sqrt{\frac{1}{15} \sum_{i=1}^{16}\left(x_{j, i}-\bar{x}_{j}\right)^{2}}$,

$$
\begin{gathered}
\hat{\beta}_{1, \text { lasso }}=\theta_{1, \text { lasso }} / s_{1}=-0.1371, \\
\hat{\beta}_{2, \text { lasso }}=\hat{\theta}_{2, \text { lasso }} / s_{2}=0.0441,
\end{gathered}
$$

and the estimate of the intercept is

$$
\bar{y}-\hat{\beta}_{1, \text { lasso }} \bar{x}_{1}-\hat{\beta}_{2, \text { lasso }} \bar{x}_{2}=64.3208
$$

so

$$
\hat{\beta}_{\text {lasso }}=\left[\begin{array}{c}
64.3208 \\
-0.1371 \\
0.0441
\end{array}\right] .
$$

Let's see how to use principal components regression with one component. Principal components regression also uses a standardized inputs and centered outputs.

First, compute the first principal component. It is an eigenvector corresponding to the largest eigenvalue of

$$
X_{s}^{t} X_{s}=\left[\begin{array}{ll}
15.00000 & 14.86635 \\
14.86635 & 15.00000
\end{array}\right]
$$


which is

$$
v_{1}=\left[\begin{array}{l}
0.7071068 \\
0.7071068
\end{array}\right] .
$$

So, the first principal component is

$$
z_{1}=X_{s} v_{1}=\left[\begin{array}{c}
-2.08919531 \\
-1.80627545 \\
-1.70005024 \\
-1.39369555 \\
-0.96150565 \\
-0.71180586 \\
-0.39559119 \\
0.06584938 \\
0.35712847 \\
0.69887111 \\
0.86450026 \\
1.27990085 \\
1.62495913 \\
1.98824550 \\
2.47606679
\end{array}\right]
$$

Then we regress $y$ on $z_{1}$ to obtain

$$
\hat{\theta}_{p c r}=\left(z_{1}^{t} z_{1}\right)^{-1} z_{1}^{t} y_{c}=2.424533
$$

Then convert back to the estimates for standardized inputs and obtain

$$
\hat{\beta}_{s, p c r}=\hat{\theta}_{p c r} v_{1}=\left[\begin{array}{c}
1.714403 \\
1.714403
\end{array}\right] .
$$


Dividing each component by the standard deviation of the corresponding explanatory variable $s_{j}=\sqrt{\frac{1}{15} \sum_{i=1}^{16}\left(x_{j, i}-\bar{x}_{j}\right)^{2}}$,

$$
\begin{aligned}
& \hat{\beta}_{1, p c r}=\hat{\beta}_{s, 1, p c r} / s_{1}=0.2464604, \\
& \hat{\beta}_{2, p c r}=\hat{\beta}_{s, 2, p c r} / s_{2}=0.0172484,
\end{aligned}
$$

and the estimate of the intercept is

$$
\bar{y}-\hat{\beta}_{1, p c r} \bar{x}_{1}-\hat{\beta}_{2, p c r} \bar{x}_{2}=29.6895
$$

so

$$
\hat{\beta}_{p c r}=\left[\begin{array}{c}
29.6895 \\
0.2465 \\
0.0172
\end{array}\right] .
$$

Now let's see how to use partial least squares regression with one component. Partial least squares regression also uses a standardized inputs and centered outputs.

First, compute the first partial least squares direction. The weights for each input are computed as

$$
\hat{\phi}_{11}=\frac{x_{1}^{t} y}{x_{1}^{t} x_{1}}=3.372861, \hat{\phi}_{21}=\frac{x_{2}^{t} y}{x_{2}^{t} x_{2}}=3.454202 .
$$


Then the first partial least squares direction is

$$
z_{1}=\hat{\phi}_{11} x_{1}+\hat{\phi}_{21} x_{2}=\left[\begin{array}{c}
-10.0908933 \\
-8.7208097 \\
-8.2152440 \\
-6.7322252 \\
-4.6343871 \\
-3.4285825 \\
-1.9052091 \\
-1.4387132 \\
0.3220939 \\
1.7292468 \\
3.3786451 \\
4.1701415 \\
6.1828069 \\
7.8449993 \\
9.5905992 \\
11.9475315
\end{array}\right] .
$$

Then we regress $y$ on $z_{1}$ to obtain

$$
\hat{\theta}_{p l s r}=\left(z_{1}^{t} z_{1}\right)^{-1} z_{1}^{t} y_{c}=0.5023084 .
$$

Then convert back to the estimates for standardized inputs and obtain

$$
\hat{\beta}_{s, p l s r}=\hat{\theta}_{p l s r}\left[\begin{array}{l}
\hat{\phi}_{11} \\
\hat{\phi}_{21}
\end{array}\right]=\left[\begin{array}{l}
1.694217 \\
1.735075
\end{array}\right] .
$$

Dividing each component by the standard deviation of the corresponding explanatory variable $s_{j}=\sqrt{\frac{1}{15} \sum_{i=1}^{16}\left(x_{j, i}-\bar{x}_{j}\right)^{2}}$,

$$
\hat{\beta}_{1, p l s r}=\hat{\beta}_{s, 1, p l s r} / s_{1}=0.24355835,
$$




$$
\hat{\beta}_{2, p l s r}=\hat{\beta}_{s, 2, p l s r} / s_{2}=0.01745637,
$$

and the estimate of the intercept is

$$
\bar{y}-\hat{\beta}_{1, p l s r} \bar{x}_{1}-\hat{\beta}_{2, p l s r} \bar{x}_{2}=29.9496
$$

so,

$$
\hat{\beta}_{p l s r}=\left[\begin{array}{c}
29.9496 \\
0.2436 \\
0.0175
\end{array}\right] \text {. }
$$




\section{CHAPTER 3 \\ SIMULATION STUDIES}

In this section, simulation studies are performed under various scenarios for regression models of the form

$$
y_{i}=\beta_{0}+\sum_{j=1}^{p} \beta_{j} x_{j, i}+\epsilon_{i}
$$

where the errors $\epsilon_{1}, \ldots, \epsilon_{n}$ are independent random variables with mean 0 and variance $\sigma^{2}$. For all of these simulations, we consider the small sample size $n=4$. We assume that these true parameter values are unknown and attempt to use each of our linear methods for regression to estimate the model and predict new responses based on observing new inputs. Since we use only a sample size of four, leave-oneout cross validation (that is, 4 -fold cross-validation) is used for each method.

Two general scenarios are considered. In Scenario 1, the main assumption is that $\beta_{1} \neq 0$ but the true values of all of the other regression coefficients are 0 , so the true model for $y$ only depends on $x_{1}$. In Scenario 2, all regression coefficients are generated from a Uniform $(-0.1,0.1)$ distribution, so the true model for $y$ is affected by all of the explanatory variables.

For all simulations, the explanatory vectors

$$
\left[\begin{array}{c}
x_{1,1} \\
\vdots \\
x_{p, 1}
\end{array}\right], \ldots,\left[\begin{array}{c}
x_{1,4} \\
\vdots \\
x_{p, 4}
\end{array}\right],
$$

are generated independently from a multivariate normal distribution with $p$-dimensional 
mean vector $[0, \ldots, 0]^{\prime}$ and covariance matrix

$$
\Sigma=\left[\begin{array}{cccccccc}
1 & \rho & \cdots & \rho & 0 & 0 & \cdots & 0 \\
\rho & 1 & \ddots & \rho & 0 & 0 & \cdots & 0 \\
\vdots & \ddots & \ddots & \vdots & \vdots & \vdots & \vdots & \vdots \\
\rho & \rho & \ddots & 1 & 0 & 0 & 0 & 0 \\
0 & 0 & \cdots & 0 & 1 & 0 & \cdots & 0 \\
0 & 0 & \cdots & 0 & 0 & 1 & \ddots & 0 \\
\vdots & \vdots & \vdots & \vdots & \vdots & \vdots & \ddots & \vdots \\
0 & 0 & \cdots & 0 & 0 & 0 & \ddots & 1
\end{array}\right] .
$$

where the upper left $G \times G$ block is 1 on the diagonal and $\rho$ off the diagonal so that there are $G-1$ covariates which are positively correlated with $x_{1}$.

In each simulation, we generate $R=1000$ data sets with $n=4$ data points

$$
\left(x_{1,1, r}, \ldots, x_{p, 1, r}, y_{1, r}\right), \ldots,\left(x_{1,4, r}, \ldots, x_{p, 4, r}, y_{4, r}\right)
$$

for $r=1, \ldots, R$ and use the 5 methods from Chapter 2 to fit the model.

New data is also simulated from the models, and the predictive performance is also compared for the five methods. Here $M=100$ new observations are generated as before

$$
\left(x_{1,1, r}^{*}, \ldots, x_{p, 1, r}^{*}, y_{1, r}^{*}\right), \ldots,\left(x_{1, M, r}^{*}, \ldots, x_{p, M, r}^{*}, y_{M, r}^{*}\right)
$$

This new data is not used to fit the model but only as test data to assess the prediction error.

Here is a description of the performance of the methods for particular choices of scenarios and parameters.

\subsection{SCENARIO 1 WITH $p=10, G=4, \rho=.1, \beta_{1}=.1$}

First, consider the simulation for this particular scenario and parameter setting. The results are compared using various measures to assess the estimates. Let 
$\hat{\beta}_{j, r}$ denote an estimate of $\beta_{j}$ based on a particular method for data set $r$. To assess the intercept, the root mean square error for the intercept

$$
\text { bORMSE }=\sqrt{\frac{1}{R} \sum_{r=1}^{R}\left(\hat{\beta}_{0, r}-\beta_{0, r}\right)^{2}}
$$

and the mean absolute error for the intercept

$$
\text { bOMAE }=\frac{1}{R} \sum_{r=1}^{R}\left|\hat{\beta}_{0, r}-\beta_{0, r}\right|
$$

are computed. The root mean square error for the coefficient of $x_{1}$

$$
\text { b1RMSE }=\sqrt{\frac{1}{R} \sum_{r=1}^{R}\left(\hat{\beta}_{1, r}-\beta_{1, r}\right)^{2}}
$$

and the mean absolute error for the coefficient of $x_{1}$

$$
\text { b1MAE }=\frac{1}{R} \sum_{r=1}^{R}\left|\hat{\beta}_{1, r}-\beta_{1, r}\right|
$$

are computed. The average root mean square error for the coefficients of the explanatory variables correlated with $x_{1}$

$$
\mathrm{bcorRMSE}=\sqrt{\frac{1}{R(G-1)} \sum_{r=1}^{R} \sum_{j=2}^{G} \hat{\beta}_{j, r}^{2},}
$$

the average mean absolute error for the coefficients of the explanatory variables correlated with $x_{1}$

$$
\mathrm{bcorMAE}=\frac{1}{R(G-1)} \sum_{r=1}^{R} \sum_{j=2}^{G}\left|\hat{\beta}_{j, r}\right|,
$$

and the mean for the largest absolute error among the coefficients of the explanatory variables correlated with $x_{1}$

$$
\mathrm{bcorMmaxE}=\frac{1}{R} \sum_{r=1}^{R} \max _{j=2, \ldots, G}\left|\hat{\beta}_{j, r}\right|
$$

are computed. The average root mean square error for the coefficients of the other explanatory variables

$$
\text { botherRMSE }=\sqrt{\frac{1}{R(p-G)} \sum_{r=1}^{R} \sum_{j=G+1}^{p} \hat{\beta}_{j, r}^{2}},
$$


the average mean absolute error for the coefficients of the other explanatory variables

$$
\text { botherMAE }=\frac{1}{R(p-G)} \sum_{r=1}^{R} \sum_{j=G+1}^{p}\left|\hat{\beta}_{j, r}\right|,
$$

and the mean for the largest absolute error among the coefficients of the the other explanatory variables

$$
\text { botherMmaxE }=\frac{1}{R} \sum_{r=1}^{R} \max _{j=G+1, \ldots, p}\left|\hat{\beta}_{j, r}\right|
$$

are computed.

To assess the predictive performance of the methods, the root mean square average prediction error

$$
\operatorname{RMSAPE}=\sqrt{\frac{1}{R} \sum_{r=1}^{R}\left(\frac{1}{M} \sum_{m=1}^{M}\left|y_{m, r}^{*}-\hat{\beta}_{0, r}-\sum_{j=1}^{p} \hat{\beta}_{j, r} x_{m . j, r}^{*}\right|\right)^{2}}
$$

the root mean square median prediction error

$$
\operatorname{RMSMPE}=\sqrt{\frac{1}{R} \sum_{r=1}^{R}\left(\operatorname{median}_{m=1, \ldots, M}\left|y_{m, r}^{*}-\hat{\beta}_{0, r}-\sum_{j=1}^{p} \hat{\beta}_{j, r} x_{m . j, r}^{*}\right|\right)^{2}}
$$

the mean absolute average prediction error

$$
\text { MAAPE }=\frac{1}{R} \sum_{r=1}^{R}\left(\frac{1}{M} \sum_{m=1}^{M}\left|y_{m, r}^{*}-\hat{\beta}_{0, r}-\sum_{j=1}^{p} \hat{\beta}_{j, r} x_{m . j, r}^{*}\right|\right)
$$

and the mean absolute median prediction error

$$
\operatorname{MAMPE}=\frac{1}{R} \sum_{r=1}^{R} \operatorname{median}_{m=1, \ldots, M}\left|y_{m, r}^{*}-\hat{\beta}_{0, r}-\sum_{j=1}^{p} \hat{\beta}_{j, r} x_{m \cdot j, r}^{*}\right|
$$

are computed.

The results for this case are given in Table 3.1 when $\sigma=.01$. As might be expected, this forward selection method is ideal for this scenario. It has the smallest error in estimating $\beta_{0}$ and $\beta_{1}$ as seen from the rows bORMSE, bOMAE, b1RMSE, and b1MAE. The LASSO is a variable selection method, it also performs relatively well 
here in estimating $\beta_{0}$ and $\beta_{1}$. The penalized methods naturally tend to have coefficient estimates near 0, so their estimation errors bcorRMSE, bcorMAE, bcorMmaxE, botherRMSE, botherMAE, and botherMmaxE are smallest. In terms of prediction error in rows RMSAPE, RMSMPE, MAAPE, and MAMPE, forward selection does best, followed by the LASSO, partial least squares, and principal components regression.

The penalty chosen for ridge regression is often very large, and in these cases the coefficient estimates are numerically equivalent to 0 and the model merely uses the mean $\bar{y}$ to model $y$. The errors reported in Table 3.1 are averages over cases where $\lambda$ is very large as well as other cases where the penalty and bias towards 0 is more moderate. In some other exploratory simulation studies (not shown here), increasing $n$ towards $p$ seems to increase the proportion of times that the penalty is moderate, but when $n<p$ in the settings we considered, cross validation selected a very large $\lambda$ for some proportion of cases.

Now, consider the same scenario and parameter setting except change $\sigma$ to .1. The results are shown in Table 3.2. With the increased noise, none of the methods should be expected to perform well. As seen in the rows b1RMSE and b1MAE, the estimation errors for forward selection, ridge regression, and the LASSO are close to or over 1 (the magnitude of $\beta_{1}$ ), while partial least squares and principal components perform better and are close to the corresponding error measurements in Table 3.1. From the rows bcorMAE and botherMAE, we see that both ridge regression and the LASSO have small estimation errors because penalization tends to keep the estimates closer to 0, while the other methods are comparable in performance with principal components performing best among the other 3 at estimating these parameters which have true values of 0 . However, when we look at the estimation error in the rows bcorRMSE, bcorMmaxE, botherRMSE, and botherMmaxE where the performance measure are based on root mean squares or maximums, we see that forward selection performs much worse. In terms of prediction error in rows 
Table 3.1-Estimation and predictive errors under scenario 1 when $p=10, G=4$, $\rho=.10, \beta_{1}=.1, \sigma=.01$.

\begin{tabular}{lrrrrr}
\hline ErrorType & forward & rr & LASSO & pcr & plsr \\
\hline bORMSE & 0.0250469 & 0.0501222 & 0.0361806 & 0.0445646 & 0.0412646 \\
bOMAE & 0.0120482 & 0.0405354 & 0.0254932 & 0.0354831 & 0.0329162 \\
b1RMSE & 0.0333462 & 0.0998462 & 0.0716465 & 0.0815382 & 0.0738318 \\
b1MAE & 0.0165709 & 0.0998438 & 0.0606491 & 0.0797003 & 0.0714885 \\
bcorRMSE & 0.0130123 & 0.0007489 & 0.0050653 & 0.0124550 & 0.0138122 \\
bcorMAE & 0.0020228 & 0.0001128 & 0.0005480 & 0.0095759 & 0.0110254 \\
bcorMmaxE & 0.0080130 & 0.0002117 & 0.0021477 & 0.0176056 & 0.0201581 \\
botherRMSE & 0.0113861 & 0.0007555 & 0.0053655 & 0.0120067 & 0.0135606 \\
botherMAE & 0.0017937 & 0.0001145 & 0.0004130 & 0.0092163 & 0.0107571 \\
botherMmaxE & 0.0080130 & 0.0002117 & 0.0021477 & 0.0176056 & 0.0201581 \\
RMSAPE & 0.0461500 & 0.0905128 & 0.0660997 & 0.0805317 & 0.0757954 \\
RMSMPE & 0.1096930 & 0.2722462 & 0.2265662 & 0.2572809 & 0.2498161 \\
MAAPE & 0.0267904 & 0.0894460 & 0.0565196 & 0.0789746 & 0.0741711 \\
MAMPE & 0.0231221 & 0.0771016 & 0.0486884 & 0.0680413 & 0.0637530 \\
\hline
\end{tabular}


Table 3.2-Estimation and predictive errors under scenario 1 when $p=10, G=4$, $\rho=.10, \beta_{1}=.1, \sigma=.1$.

\begin{tabular}{lrrrrr}
\hline ErrorType & forward & rr & LASSO & pcr & plsr \\
\hline bORMSE & 0.1380031 & 0.0709881 & 0.0713622 & 0.0710826 & 0.0729300 \\
bOMAE & 0.0843366 & 0.0560576 & 0.0558694 & 0.0562169 & 0.0576135 \\
b1RMSE & 0.1111229 & 0.0998317 & 0.0987464 & 0.0828913 & 0.0760386 \\
b1MAE & 0.0926896 & 0.0998272 & 0.0972469 & 0.0806224 & 0.0720920 \\
bcorRMSE & 0.0640246 & 0.0009969 & 0.0104674 & 0.0184128 & 0.0246243 \\
bcorMAE & 0.0177853 & 0.0001489 & 0.0011247 & 0.0136291 & 0.0185488 \\
bcorMmaxE & 0.0659735 & 0.0002522 & 0.0042869 & 0.0250698 & 0.0341537 \\
botherRMSE & 0.0803457 & 0.0009950 & 0.0101962 & 0.0181386 & 0.0241939 \\
botherMAE & 0.0174658 & 0.0001463 & 0.0011250 & 0.0132715 & 0.0182018 \\
botherMmaxE & 0.0659735 & 0.0002522 & 0.0042869 & 0.0250698 & 0.0341537 \\
RMSAPE & 0.2382439 & 0.1280873 & 0.1298436 & 0.1275988 & 0.1312255 \\
RMSMPE & 0.3619337 & 0.3227902 & 0.3235847 & 0.3224913 & 0.3255234 \\
MAAPE & 0.1901731 & 0.1263774 & 0.1274267 & 0.1256517 & 0.1288356 \\
MAMPE & 0.1630916 & 0.1086849 & 0.1095212 & 0.1078740 & 0.1107993 \\
\hline
\end{tabular}

RMSAPE, RMSMPE, MAAPE, and MAMPE, principal components regression does best, but it is only slightly better than ridge regression. Here, as in the previous simulation, ridge regression is very close to merely using the mean $\bar{y}$ to model $y$. The other methods do worse or about the same as ridge regression so it appears that the none of the methods are able to effectively use information from the inputs $x_{1}, \ldots, x_{10}$ to predict the output $y$.

Figure 3.1 plots the values of b1MAE and MAAPE as $\sigma$ changes from .01 to .10 holding the other true parameter values constant. The left panel illustrates the 
estimation error b1MAE and the right panel illustrates the prediction error MAAPE. When $\sigma$ is small, forward selection does best at estimation and prediction followed by the LASSO. As $\sigma$ increases, the estimation error for forward selection and the LASSO become worse. The prediction error for forward selection in particular is very poor when $\sigma$ becomes large, and the prediction errors for the other methods are similar when $\sigma$ is large.

\subsection{SCENARIO 1 WITH $G=4, \rho=.1, \beta_{1}=.5, \sigma=.1$}

Next, we study the effect of increasing the number of explanatory variables $p$ under scenario 1 with these settings for the other parameters. Figure 3.2 plots the values of b1MAE and MAAPE as $p$ changes from 10 to 100 holding the other parameters constant. The left panel illustrates the estimation error b1MAE and the right panel illustrates the prediction error MAAPE. As $p$ increases, both the estimation and prediction errors tend to increase for all methods. Forward selection has much smaller estimation error for $\beta_{1}$ but its prediction error is poor once $p$ exceeds around 70. For the estimation error, principal components regression, partial least squares regression, and the LASSO are close, with the LASSO doing best among these three methods when $p$ is large. For the prediction error also, principal components regression, partial least squares regression, and the LASSO are close, though the LASSO is best among these three methods over the range of $p$. Under these settings, partial least squares regression is slightly better than principal components regression in terms of estimation of $\beta_{1}$ and prediction.

\subsection{SCENARIO 2 WITH $p=10, G=4, \rho=.1$}

Now, some simulations under Scenario 2 are considered. Under this scenario, all regression coefficients are nonzero so the mean for the true model is a linear 
combination of all $p$ variables even though there are only 4 observations. To assess the estimation error, we redefine the average mean absolute error for the coefficients of the correlated explanatory variables as

$$
\text { bcorMAE }=\frac{1}{R G} \sum_{r=1}^{R} \sum_{j=1}^{G}\left|\hat{\beta}_{j, r}-\beta_{j}\right|,
$$

and the average mean absolute error for the coefficients of the other explanatory variables as

$$
\text { botherMAE }=\frac{1}{R(p-G)} \sum_{r=1}^{R} \sum_{j=G+1}^{p}\left|\hat{\beta}_{j, r}-\beta_{j}\right| .
$$

Figure 3.3 plots the values of bcorMAE and MAAPE as $\sigma$ changes from .01 to .10 holding the other parameters constant. The left panel illustrates the estimation error bcorMAE and the right panel illustrates the prediction error MAAPE. The plot for botherMAE has similar properties to the plot for bcorMAE so it is not shown here. The estimation error depends on the overall variance, but overall, the estimation error is smallest for ridge regression, followed by the LASSO, principal components regression, partial least squares regression, and forward selection which is much worse. As $\sigma$ increases, the prediction error for each method increases. Overall, the derived input methods do best in terms of prediction, with partial least squares being slightly better. The penalized methods are similar in prediction error, with ridge regression being slightly better than the LASSO. The prediction error for forward selection is much higher than all of the other methods.

\subsection{SCENARIO 2 WITH $G=4, \rho=.1, \sigma=.01$}

Next, we study the effect of increasing the number of explanatory variables $p$ under scenario 2 with these settings for the other parameters. Figure 3.4 plots the values of bcorMAE and MAAPE as $p$ changes from 5 to 50 holding the other parameters constant. The left panel illustrates the estimation error bcorMAE and the right panel illustrates the prediction error MAAPE. The plot for botherMAE has 
similar properties to the plot for bcorMAE so it is not shown here. The estimation error depends on the overall variance, but overall, the same ordering occurs as in the previous simulation under Scenario 2: the estimation error is smallest for ridge regression, followed by the LASSO, principal components regression, partial least squares regression, and forward selection. Also, as $p$ increases, the prediction error for each method increases, and the same ordering for predictive error occurs as in the previous simulation under Scenario 2. The derived input methods do best, with partial least squares being slightly better, then the penalized methods are next, with ridge regression being slightly better, and the prediction error for forward selection is much higher than the other methods. 
Figure 3.1 - Estimation and predictive errors under scenario 1 when $p=10, G=4$, $\rho=.10, \beta_{1}=.1$
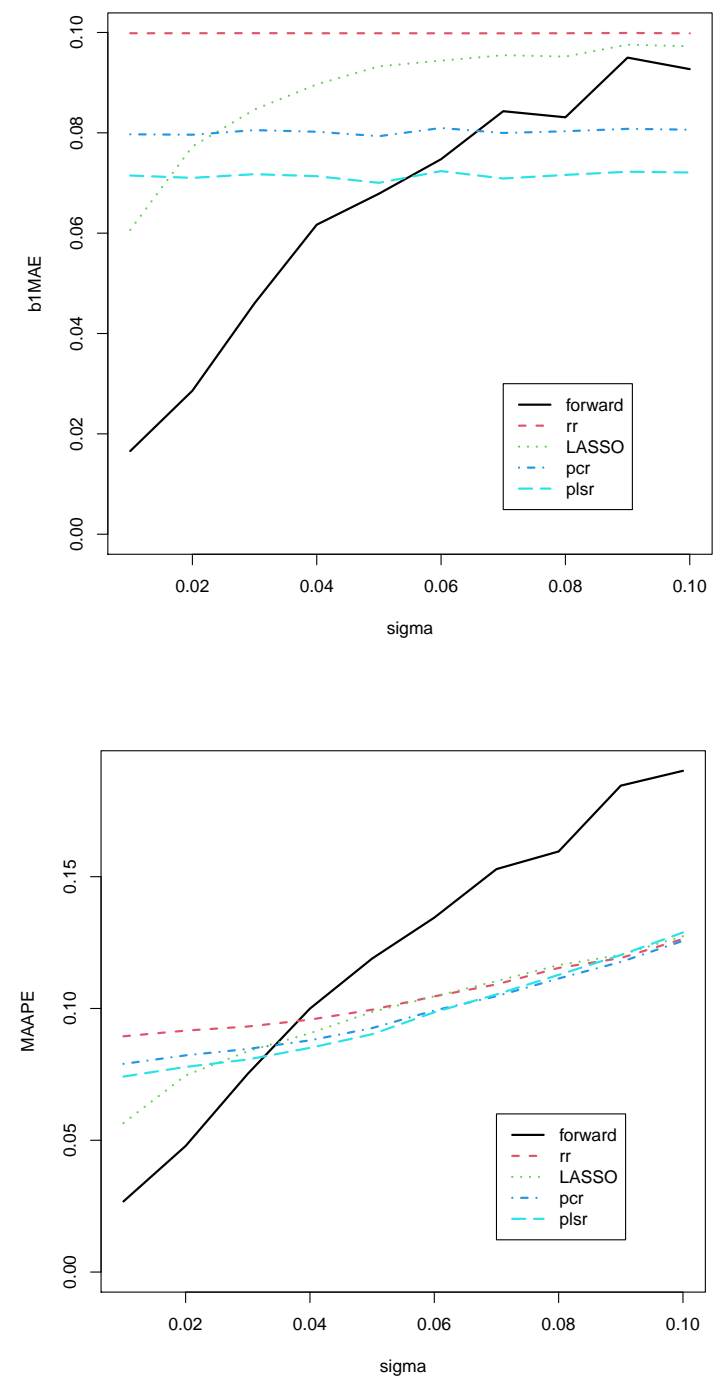
Figure 3.2-Estimation and predictive errors under scenario 1 when $G=4, \rho=.1$, $\beta_{1}=.5, \sigma=.1$
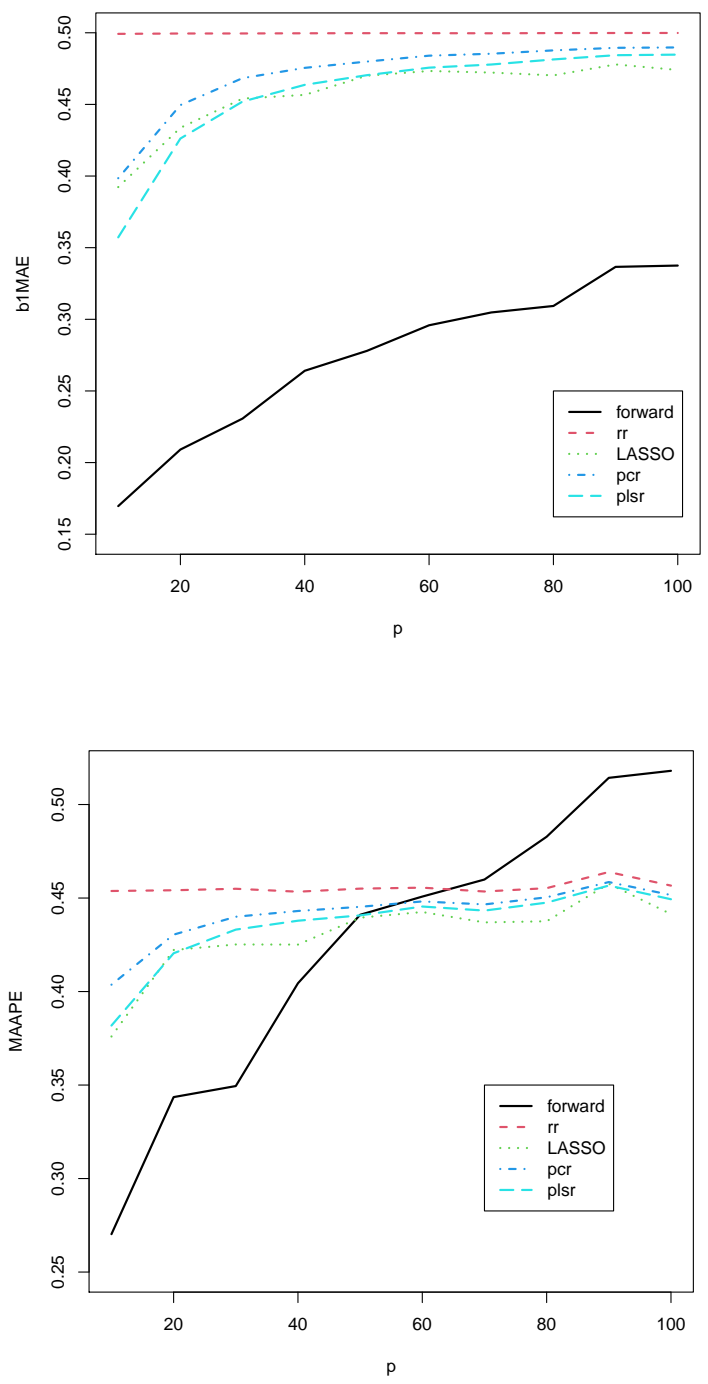
Figure 3.3-Estimation and predictive errors under scenario 2 when $p=10, G=4$, $\rho=.10$
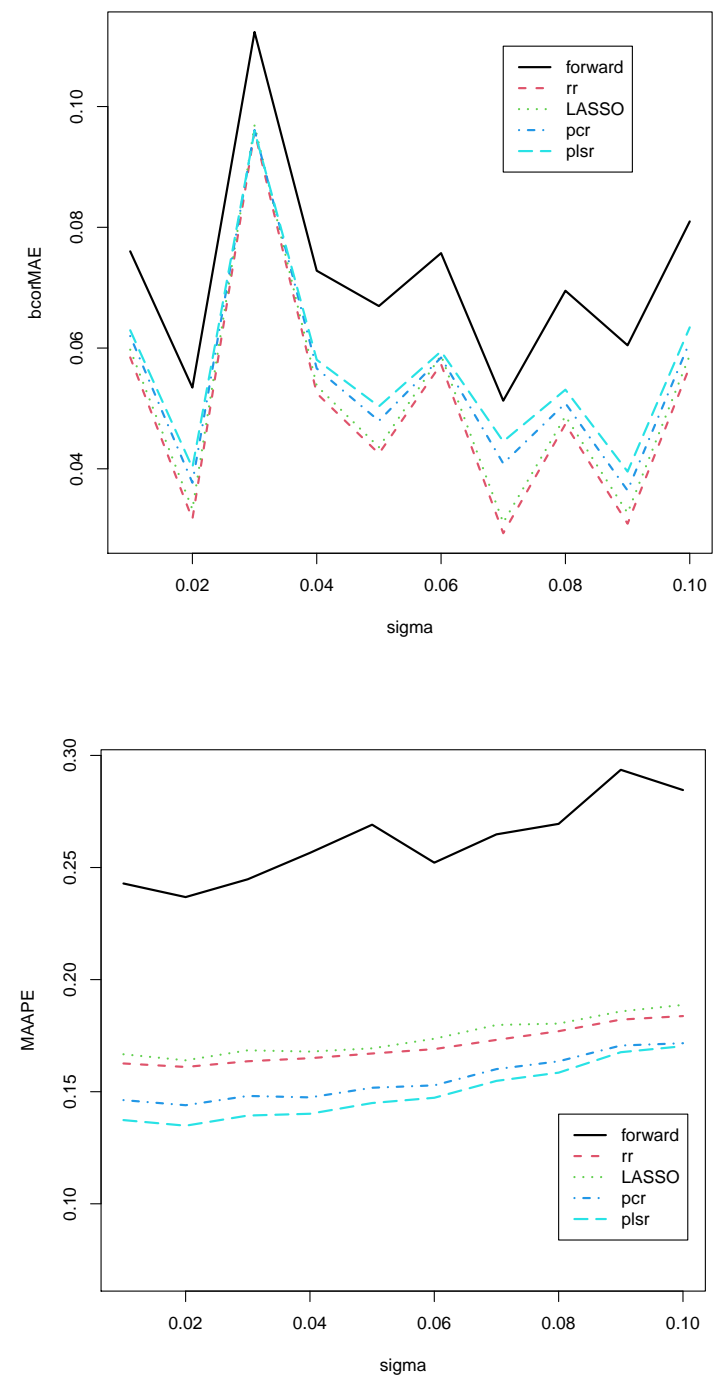
Figure 3.4-Estimation and predictive errors under scenario 2 when $G=4, \rho=.10$, $\sigma=.01$
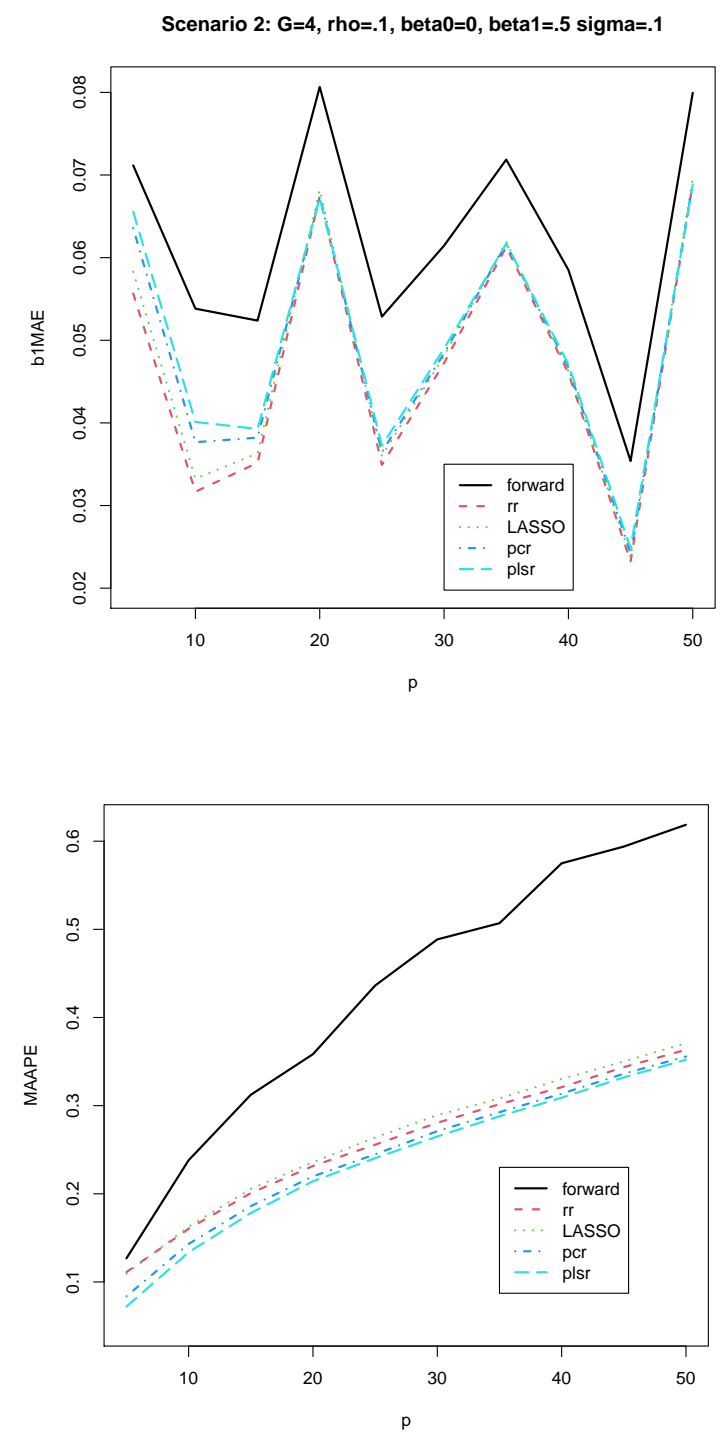


\section{CHAPTER 4}

\section{REAL DATA}

In chapter 2, we have discussed five methods - Forward selection, Ridge Regression, LASSO, Principal Component Regression (pcr) and Partial Least Squares Regression (plsr). Now we will apply these methods to a real data set to build a model to predict the weights of mice based on microarray expression data for a large number of genes.

Software tools: The project is entirely handled by RStudio from preprocessing to building the model and prediction with the help of $\mathrm{R}$ libraries glmnet [8] and $p l s[13]$.

\subsection{DATASET BACKGROUND AND PREPROCESSING}

Dataset Description: The data set we used was downloaded from tutorial IV at [3]. This data is in the file "BluemoduleGenesWeightandSNP.csv". It originally appeared in [5] and was later also analyzed in [6]. It has 536 row fea-

ture variables in the rows with gene symbols Scd1, Ppic, Anax2,..., Abhd3, and 158 columns including mice with unique id F22, F23, F214, F215, . , F2357 with Weight representing the weight of the mice.

Preprocessing: We are only interested in seeing how the 5 methods that we are studying can use the gene expression data available to predict the mouse weights so we delete some rows and columns with other information that we will not use. Then we delete some columns corresponding to mice with missing weights. 
Afterwards we deleted rows for genes with missing expression values. Then in $\mathrm{R}$ read the weights into a vector $y$ and the gene expressions into $X$, which is our design matrix. The $X$ is the transpose of how it appears in Excel so the the gene expressions are in the columns and mice are in the rows. After preprocessing, $X$ is a $132 \times 434$ design matrix and $y$ is a 132-dimensional vector.

\subsection{MODEL BUILDING AND RESULTS}

After setting all the random seed to 123456, we divide the data using a 70-30 spilt for training and testing sets, respectively. Then we apply each method and predict the weight of mice based on each method. We assess the performances of each method using the evaluation metrics root mean square error

$$
R M S E=\sqrt{\frac{S S E}{n}},
$$

mean absolute error

$$
M A E=\frac{1}{n} \sum_{i=1}^{n}\left|\hat{y}_{i}-y_{i}\right|,
$$

and the R-squared statistic

$$
R^{2}=1-\frac{S S E}{S S T}
$$

where $S S E=\sum_{i=1}^{n}\left(\hat{y}_{i}-y_{i}\right)^{2}$ and $S S T=\sum_{i=1}^{n}\left(y_{i}-\bar{y}\right)^{2}$.

Forward Selection: We know that the stepwise regression (or stepwise selection) consists of iteratively adding and removing predictors, in the predictive model, in order to find the subset of variables in the data set resulting in the best performing model, that is a model that lowers prediction error. Forward selection, which starts with no predictors in the model, iteratively adds the most contributive predictors, and stops when the improvement is no longer statistically significant [1]. The RMSE, MAE and R-squared values for the ridge regression model on the training data are $0.005303152,0.004285677$ and 0.9999992 , respectively. For 
the test data, the results for these metrics are 5.966883, 4.688417 and 0.08540092 , respectively.

\section{Ridge Regression:}

Ridge regression is an extension of linear regression where the loss function is modified to minimize the complexity of the model. This modification is done by adding a penalty parameter that is equivalent to the square of the magnitude of the coefficients. One of the major differences between linear and regularized regression models is that the latter involves tuning a hyperparameter, lambda. The code runs the glmnet() model several times for different values of lambda. We can automate this task of finding the optimal lambda value using the cv.glmnet() function.

The optimal lambda value comes out to be 3.162278 and will be used to build the ridge regression model. We will also create a function for calculating and printing the results, which is done with the eval_results() function. The next step is to use the predict function to generate predictions on the train and test data. Finally, we use the eval_results() function to calculate and print the evaluation metrics. The RMSE, MAE and R-squared values for the ridge regression model on the training data are $1.306087,1.041384$ and 0.9519684 , respectively. For the test data, the results for these metrics are 3.541635, 2.691895 and 0.6777868 , respectively.

\section{LASSO:}

Lasso regression, or the Least Absolute Shrinkage and Selection Operator, is also a modification of linear regression. In lasso, the loss function is modified to minimize the complexity of the model by limiting the sum of the absolute values of the model coefficients , also called the 11 -norm.

Using an l1-norm constraint forces some weight values to zero to allow other coefficients to take non-zero values. The first step to build a lasso model is to find the optimal lambda value. The output is the best cross-validated lambda, which comes out to be 0.1584893 . Once we have the optimal lambda value, we train 
the lasso model. The RMSE, MAE and R-squared values on the training data are $1.764331,1.39817$ and 0.9123519 , respectively. The results on the test data are $3.544719,2.772592$ and 0.6772255 , respectively.

\section{Principal Component Regression (PCR):}

A possible drawback of PCR is that we have no guarantee that the selected principal components are associated with the outcome. Here, the selection of the principal components to incorporate in the model is not supervised by the outcome variable. Our analysis shows that, choosing 81 principal components $($ ncomp $=81$ ) gives the smallest prediction error RMSE. The RMSE, MAE, and R-squared values on the training data are $2.153796,1.681829$ and 0.8693854 , respectively. The results on the test data are $3.523516,2.806547$ and 0.6810752 , respectively.

Partial Least Squares: An alternative to PCR is the Partial Least Squares (PLS) regression, which identifies new principal components that not only summarizes the original predictors, but also that are related to the outcome. These components are then used to fit the regression model. So, compared to PCR, PLS uses a dimension reduction strategy that is supervised by the outcome.

Like PCR, PLS is convenient for data with highly-correlated predictors. The number of principal components used in PLS is generally chosen by cross-validation. Predictors and the outcome variables should be generally standardized, to make the variables comparable. The RMSE, MAE, and R-squared values on the training data are $1.853102,1.519636$ and 0.9033101 , respectively. The results on the test data are $3.627071,2.808874$ and 0.6620536 , respectively.

\subsection{COMPARISON OF RESULTS}

Looking at the results for the test data, Ridge Regression, LASSO, Principal Components Regression, and Partial Least Squares Regression all perform similarly 
in terms of RMSE, MAE, and R-squared measures while Forward Selection performs much worse based on all measures. Principal Components Regression is best in terms of RMSE and R-squared, while Ridge Regression is best in terms of MAE. The training errors show that Forward Selection produces an almost perfect fit, but this just shows that the method is overfitting the data. 


\section{REFERENCES}

[1] P. Bruce and A. Bruce, Practical Statistics for Data Scientists, O'Reilly Media, Inc, 127-172, 2017.

[2] C.L. Mallows, Some comments on $C_{P}$, Technometrics, 15(4), 661-675, 1973.

[3] Data and Statistical $R$ Code: Integrating Genetics and Network Analysis to Characterize Genes Related to Mouse Weights, https://horvath.genetics. ucla.edu/html/CoexpressionNetwork/MouseWeight/, November 12, 2008.

[4] B. Efron, T. Hastie, I. Johnstone and R. Tibshirani, Least angle regression, The Annals of Statistics, 32, 407-499, 2004.

[5] A. Ghazalpour, S. Doss, B. Zhang, S. Wang, C. Plaisier, R. Castellanos, A. Brozell, E.E. Schadt, T.A. Drake, A.J. Lusis, S. Horvath S, Integrating genetic and network analysis to characterize genes related to mouse weight, PloS Genetics 2(8), 2006.

[6] R. Gill, S. Datta and S. Datta, A statistical framework for differential network analysis from microarray data, BMC Bioinformatics 11, 95, 2010.

[7] T. Hastie, R. Tibshirani and J. Friedman, The Elements of Statistical Learning: Data Mining, Inference, and Prediction, 2nd edition, Springer-Verlag, 2009.

[8] T. Hastie, R. Tibshirani and J. Friedman, Regularization Paths for Generalized Linear Models via Coordinate Descent, Journal of Statistical Software 331, 2010. 
[9] S. Hettigoda, Computation of Least Angle Regression Coefficient Profiles and LASSO estimates, M.A. Thesis, University of Louisville, 2016.

[10] Z. Lau, Applications of the Generalized Cross Validation Statistic, M.A. Thesis, University of Louisville, 2007.

[11] D.C. Lay, Linear Algebra and its Applications, 4th edition, Addison-Wesley, 2011.

[12] J.W. Longley. An appraisal of least-squares programs from the point of view of the user, Journal of the American Statistical Association 62, 819-841, 1967.

[13] B.-H. Mevik, R. Wehrens and K.H. Liland, pls: Partial Least Squares and Principal Component Regression. R package version 2.7-2., https://CRAN. R-project.org/package=pls, 2019.

[14] A.C. Rencher and B. Schaalje, Linear Models in Statistics, 2nd edition, Wiley, 2008.

[15] G.A.F. Seber and A.J. Lee, Linear Regression Analysis, 2nd edition, Wiley, 2003.

[16] Z. Zhang. Variable selection with stepwise and best subset approaches, Annals of Translational Medicine, 4 (7), 136, 2016. 


\title{
CURRICULUM VITAE
}

\author{
Rajesh Sikder \\ 2501 S 4th St \\ Louisville, KY 40208 \\ (206) 369-4585 \\ rajeshsikder@ymail.com
}

\section{EDUCATION}

University of Louisville, Louisville, KY

Master of Arts, Mathematics; August 2020

Khulna University, Khulna, Bangladesh

Bachelor of Science, Mathematics; February 2015

\section{TECHNICAL SKILLS}

Programming Languages: Python, R, SAS

Other Tools/Technologies: PostgreSQL, TensorFlow, Excel, Power BI, Weka, LaTeX, Mathematica, SPSS

\section{TEACHING EXPERIENCE}

Graduate Teaching Assistant, University of Louisville; 2017-2020 